\title{
COMPARACIÓN DE LA DIVERSIDAD FAUNÍSTICA (HERPETOFAUNA) EN CACAOTALES Y RASTROJOS, SIUNA 2011
}

\author{
Edwin Antonio Rodríguez Leiva ${ }^{[1]}$ \\ Yoicelin Nohemí Jiménez Romero ${ }^{[2]}$ \\ Efraín de Jesús Peralta Tercero ${ }^{[3]}$ \\ Oscar Montalván Castellón ${ }^{[4]}$
}

\section{Resumen}

La investigación ha permitido describir la diversidad faunística (herpetofauna) en cacaotales y rastrojos, Siuna- 2011, en 15 unidades productivas, distribuidas en 5 comunidades. Se caracterizaron plantaciones de cacao y rastrojos para determinar la diversidad de herpetofauna, mediante un estudio cuantitativo-observacional-comparativo. Los resultados muestran: las tipologías de cacao multiestratos con 5 representaciones, cacao simple con 8 y cacao con musáceas 2. En rastrojos de 1 a 5 años 11 representaciones, de 6 a 10 años 1 representación y de 11 a 15 años 3. Las especies de reptiles encontradas fueron: 197 individuos agrupados en 24 especies y 10 familias y 17 géneros en todas las tipologías de cacao. En rastrojos menores a 5 años se encontraron 12 especies y 30 individuos que representan el $51.72 \%$. En cacao y rastrojos no hay diferencias entre hábitat para las especies de herpetofauna, debido a que las parcelas muestreadas se encontraban una contiguo a la otra. El índice de diversidad dio valores de mediana diversidad $(2,368-2,236)$ en ambos sitios de alta diversidad tanto en cacaotales y rastrojos superiores a o.66 para Simpson 1-D.

Palabras clave: Anfibios; reptiles; composición de bosque; habitat; especies; tipologías; biodiversidad.

\section{Summary}

The research has described the faunal diversity (herpetofauna) in cacao plantations and stubble, in the municipality of Siuna, year 2011, in 15 productive units distributed in 5 communities. Cacao and stubble plantations were characterized in order to determine the diversity of herpetofauna, through a quantitative-observational-comparative study.

The results showed different typologies: multilayer cacao: 5 representations; simple cacao: 8 , and cacao with musaceas: 2 . Related to stubble from 1-5 years there are 11 representations; from 6 to 10 years 1 representation and $11-15$ years 3 representations.

\footnotetext{
[1] Ingeniero Agroforestal. Correo: edwin.rodriguez1987@yahoo.com

[2] Ingeniera Agroforestal. Correo: ingafyoice@gmail.com

[3] Máster en Docencia Universitaria, docente de la URACCAN. Correo: eparaltat11@hotmail.com

[4] Máster en Docencia Universitaria, Técnico IREMADES. Correo: montesiuna@yahoo.com. Asesor.
} 
The reptile species found were: 197 individuals grouped into 24 species and 10 families, as well as 17 types of cacao.

In stubbles under 5 years, 12 species and 30 individuals were found representing $51.72 \%$. In cacao and stubbles there is no difference between habitats for herpetofauna species, due to the fact that the sampled plots were contiguous one with the other. The diversity index gave median values diversity (2.368 to 2.236) in both sites of high diversity, both in the cacao and stubbles above 0.66 for 1-D Simpson.

Keywords: Amphibians; reptiles; forest composition; habitat; species; typologies; biodiversity.

\section{Introducción}

En Latinoamérica, los procesos de degradación ecológica son producto de vertiginosos cambios en el uso del suelo, el medio ambiente, asociados al aumento de la población humana. En este contexto la destrucción, la fragmentación y la simplificación de hábitats naturales, se perfilan como las principales causas en la pérdida de la biodiversidad, actúan sinérgicamente sobre las especies con poblaciones reducidas o amenazadas.

Nicaragua por su condición geográfica es uno de los países con mayor diversidad biológica de la región centroamericana. Los anfibios y reptiles son sólo un grupo de animales que forman parte de esa riqueza biológica que posee nuestro país. A la fecha se han reportado 248 especies de reptiles y anfibios que están debajo de los reportes de países vecinos (Honduras con 300 especies y Costa Rica con 360), lo que puede afirmarse que estos son grupos zoológicos menos conocidos en Nicaragua.

En esta investigación se compara la diversidad faunística (herpetofauna) en cacaotales y rastrojos en 5 comunidades del municipio de Siuna, 2011. Se caracterizan las diferentes plantaciones de cacao y tipos de rastrojos, segun las condiciones ambientales y estructurales, se describen las especies de fauna silvestre (anfibios y reptiles) y se determinan los índices de diversidad faunística en cacaotales y rastrojos.

El documento sirve como base de datos para la toma de decisiones en el aprovechamiento y cuido de la fauna silvestre por parte de los comunitarios, instituciones de la administración pública y como fuente de información a personas que estén interesadas en profundizar la temática sobre la diversidad faunística de nuestra región.

\section{Diseño metodológico}

Ubicación del estudio: comunidades de El Carao, el Come Negro, el Guayabo, Rosa Grande, Floripón. Tipo de estudio: es cuantitativo, observacional y comparativo. Universo de estudio: está conformado por todos los sistemas agroforestales (cacaotales 
y rastrojos) presentes en el trópico húmedo. Muestras: Comunidades El Carao, Come Negro, El Guayabo, Rosa Grande y Floripón. Unidad de análisis. Diferentes especies de anfibios y reptiles (herpetofauna) que se encontraron en el área. Variables: Especie de fauna silvestre encontrada en cacaotales y rastrojos. c) Índice de diversidad faunística. d) Riqueza específica. f) Densidad poblacional.

\section{Técnicas e instrumentos para la recolección de información}

Observación directa: Esta técnica se aplicó para reconocimiento del área y caracterización sobre tipologías de plantaciones de cacao, además en base a la información que generó el productor en cuanto a la edad de las áreas de rastrojos sin intervención.

Inventario de herpetofauna: Esta técnica consistió en inventariar los individuos de diferentes especies que se encontraron en las dos comunidades (cacaotales y rastrojos) para la identificación de la diversidad faunística (herpetofauna) a través de observaciones directas.

Entrevistas individuales: Se utilizaron para definir en qué tipologías se encontraban las plantaciones de cacao como el área de rastrojos; el productor domina la edad de sus tierras en descanso, que fue un parámetro de importancia a conocer en el estudio, mientras que en cacao se determinó por la observación actual.

\section{Resultados y discusión}

Caracterización de plantaciones de cacao y tipos de rastrojos en las 5 comunidades en estudio, se encontraron 5 sistemas Cacao-Multiestratos, 8 sistemas Cacao-Simple y 2 sistemas Cacao-Musácea.

Cacao-Multiestratos: fueron encontrados en 4 de las 5 comunidades, los que poseían una estructura vertical de 3-4 estratos establecido, café en el primero, cacao y musácea en el segundo estrato, árboles frutales en el tercero y maderables en el último estrato de hasta 20 metros de altura. En la estructura horizontal, el cacao se encuentra establecido a un distanciamiento de $4 \mathrm{~m} \mathrm{x} 4 \mathrm{~m}$ para una densidad poblacional de aproximadamente 625 plantas/manzanas, el estrato conformado por café (Coffea arábiga) tiene un distanciamiento de $2 \mathrm{~m} \mathrm{x} 4 \mathrm{~m}$, mientras que los frutales, maderables cítricos no tienen un arreglo espacial bien definido.

Cacao simple: se encontraron conformados por un estrato bien definido (cacao); seguido por árboles dejados de forma natural una vez botada la mayor parte de vegetación con el principal objetivo de proporcionarle sombra permanente al cultivo, además de algunos beneficios adicionales (leña y madera). 
Los sistemas cacao-musácea: se encontraron en menor proporción debido a que son plantaciones jóvenes que están iniciando a producir y presentan alta densidad de musácea para sombra al cultivo, mientras otros árboles de sombra permanentes se desarrollan (Soto, 2009 y Matey et al, 2010). Encontraron diferentes tipologías de cacao y los agruparon de acuerdo a las condiciones ambientales y estructurales que poseía cada uno de ellos (estratos horizontales - verticales y sombra), estos resultados son similares a los encontrados en las comunidades donde se realizó la investigación. Cuadro No. 1. Los resultados obtenidos en cuanto a la caracterización de cacao, son similares a la investigación realizada por Soto (2009) otro estudio realizado por Matey et al, (2010). En donde encontraron diferentes tipologías de cacao y los agruparon de acuerdo a las condiciones ambientales y estructurales que poseía cada uno de ellos (estratos horizontales - verticales y sombra). Los cuales fueron agrupados para estimar la heterogeneidad de la estructura vertical - horizontal de las plantaciones de cacao como los fragmentos de rastrojos.

\section{Caracterización del rastrojo}

Para el caso de los rastrojos se analizaron tres categorías de acuerdo a edades. De 1-5 años, de 6-10 años y de 11-15. En las categorías de 1-5 años se encontraron 11 parcelas, 1 en las de 6-10 y 3 en la de 11-15 años.

Cuadro No. 1. Tipologías de las plantaciones de cacao y rastrojos encontradas, de acuerdo con las condiciones ambientales y estructurales dentro del proyecto ICCO

\section{M=Multiestratos, S simple, MS, Musáceas, R1 = Rastrojos de 1-5 años, R2= Rastrojos de 6-}

\begin{tabular}{|c|c|c|c|c|c|c|}
\hline \multirow{2}{*}{ Comunidades } & \multicolumn{6}{|c|}{ Tipologías de cacao y rastrojos } \\
\hline & Cacao M & Cacao S & Cacao MS & $\mathbf{R} 1$ & $\mathbf{R} 2$ & $\mathbf{R}_{3}$ \\
\hline Come Negro & 1 & 2 & o & 2 & $\mathrm{O}$ & 1 \\
\hline Carao & 1 & 2 & 0 & 3 & 0 & 0 \\
\hline Guayabo & 2 & 1 & 0 & 3 & 0 & 0 \\
\hline Rosa Grande & 0 & 2 & 1 & 2 & 0 & 1 \\
\hline Floripón & 1 & 1 & 1 & 1 & 1 & 1 \\
\hline Total & 5 & 8 & 2 & 11 & 1 & 3 \\
\hline
\end{tabular}

\section{Diversidad de anfibios en cacao y en rastrojo}

Se identificaron 23 individuos de anfibios, distribuidos en las tipologías de cacao y rastrojos de la siguiente forma: 3 en cacao con musáceas, 8 en cacao simple, 2 en rastrojos de 1-5 años, 1 en rastrojos de 5-10 años y 1 en rastrojos de 10-15 años. Las especies encontradas fueron 8 de la clase Amphybia, un individuo no identificado, 7 individuos de Bufus marinus, 1 de Chaunus marinus, 3 de Leptodactylus melanonotus. 
Se encontraron 4 especies de anfibios en rastrojos, como las especies Smilisca phaeota, Phrynohyas venulosa eIncilius coniferus, comparten los hábitat tanto cacao como rastrojos. Se encontró 1 especie ( $25 \%$ ) en rastrojos de 15 - 10 años, 1 especie (25\%) en rastrojos de $10-5$ años y 2 especies (50\%) en rastrojos menores a 5 años.

Para las tipologías estructurales y ambientales en cacao se distribuyeron: 3 especies (37.5\%) registradas en cacao multi estrato, 5 especies (62.5\%) encontradas en cacao simple, no se registraron especies en cacao musácea.

\section{Diversidad de reptiles en cacao y en rastrojo}

Se encontraron 197 individuos agrupados en 24 especies, correspondientes a 10 familias, representando a 17 géneros del orden Squamata (serpientes y lagartijas) de la clase Reptilia. De ellas 5 especies como Basiliscus vittatus, Norops sericeus, Norops humilis, Slender anole y Ameiva festiva comparten los dos hábitat, y encontrándose de manera consecutivas por cada una de las tipologías. En cambio el Norops spp, que se encontró solamente en rastrojos.

Con relación a las diferentes tipologías de Cacao Se encontraron un total de 23 especies de reptiles; 8 especies en Cacao Multi estrato, 13 especies en Cacao Simple y 2 especies en Cacao Musácea.

Las especies encontradas por tipología en rastrojos fue: 6 especies (33.33\%) en rastrojos de 15- 10 Años, 2 especies (11.11\%) en rastrojos de 10-5 Años y 10 especies (55.56\%) en rastrojos menores a 5 Años.

\section{Riqueza especifica}

La riqueza específica (alfa) para la comunidad de cacao según Margalef fue de 4.13 y para rastrojo fue de 4.19. Para la complementariedad calculada según la ecuación de Whittaker (1972) refleja que el $75 \%$ de las especies comparten hábitat entre cacao y rastrojo, lo que indica que estas especies no son exclusivas de un solo hábitat. Un $25 \%$ (8) del total de las 32 especies encontradas no comparten o se encuentran en un solo sitio.

En total se encontraron 32 especies, se registraron 22 en cacao y 18 en rastrojos. Para un total de 219 individuos, encontrándose 160 en cacao y 59 en rastrojos. De esta manera se confirma la hipótesis que: "Los cacaotales proporcionan mejores condiciones ambientales para la herpetofauna que los rastrojos". 


\section{Índices de diversidad para cacao y rastrojos en general.}

En el estudio se obtuvieron datos para cacaotales y rastrojos entre los valores (1.6 a 3.0) para categorizarlos como índices de mediana diversidad. Este concuerda Shannon \& Hidalgo. J. (2009) refleja que el rango 1.6 a 3.o pueden ser considerados como sitios de mediana diversidad en su investigación.

Pero difiere a un estudio realizado en Lojas ecuador por Hidalgo. J. (2009) en el que obtuvo resultados bajos (o.85) en bosques secundarios y o.99 en sistemas agroforestales de diversidad (Shannon), difieren en comparación a nuestro estudio. Porque se encontró que la diversidad según Shannon toma un valor de diversidad media para los dos sitios $(2.368-2.236)$.

El número de individuos y especies encontrados tanto en el cacao como en rastrojos fueron similares, este resultado difiere con Hidalgo. J. (2009), el número de especies como de individuos fueron muy diferentes en bosques secundarios como en sistemas agroforestales con cacao.

Para el caso de Simpson se obtuvieron valores de alta diversidad tanto en cacaotales como en rastrojos, los cuales se encontraron en el rango superior de o.66, datos que reflejan que las dos comunidades presentan dominancia en los índices de diversidad de forma general.

Los índices de diversidad (Shannon \& Simpson) son similares en las dos comunidades, se debe principalmente a que las parcelas de cacao y los parches de rastrojos se encuentran juntos en las unidades productivas y que puede decirse que sirven de corredores, habitat. Esto reflejado en ambas comunidades (Cacao y Rastrojos) se encontraron individuos de las mismas especies.

\section{Densidad poblacional de anfibios y reptiles en Cacaotales y Rastrojos}

La densidad poblacional general se obtuvieron los siguientes: 44.62 individuos/ha para rastrojos y 54.58 individuos/ha para cacao. Las especies reptiles como la Ameiva festiva presentan densidades más altas de 8 individuos/ha en plantaciones de Cacao Simple y en Rastrojos de 10-15 años, seguidos por el Norops humiles y el Norops sericeus con una densidad poblacional de 5 individuos/ha.

La densidades más bajas de los reptiles son: Basiliscus Basiliscus, el Basiliscus plumifrons, el Norops oxylophus, el (Norops spp), la Mabuya unimarginata, el Sphaerodactylus millepunetatus, la Leptophis nebulosus, la Imantodes cenchoa y el Micrurus nigrocinctus, (Spolotes pullatus), (Clelia clelia), (Botrox asper), todas estas especies con 1 individuo/ ha. Con respecto a los anfibios, el Bufus marinus es la especie que sobre sale con 2 individuos/ha, seguidos por las demás especies con un solo individuo. 
Cuadro No. 2. Especies de anfibios y reptiles encontrados en diferentes tipos de plantaciones de cacao y rastrojo

Anfibios

\begin{tabular}{|c|c|c|c|c|c|c|c|c|}
\hline \multirow{2}{*}{ Clase } & \multirow{2}{*}{ N. común } & \multirow{2}{*}{ N. científico } & \multicolumn{6}{|c|}{ Lugar donde se encontró } \\
\hline & & & CM & CS & CMS & R1 & $\mathbf{R} \mathbf{2}$ & $\mathbf{R}_{\mathbf{3}}$ \\
\hline \multirow{8}{*}{ Anfibio } & Sapo gigante & Chaunus marinus & & & & 1 & & 1 \\
\hline & Sapo gigante & Bufus marinus & & 3 & & & & \\
\hline & $\begin{array}{l}\text { Sapito Tungara o rani- } \\
\text { ta del charco }\end{array}$ & leptodactylus melanonotus & & 2 & & & & \\
\hline & Sapito arbóreo & Incilius coniferus & 1 & & & & & 1 \\
\hline & Sapo & Incilius melanochlorus & 1 & 1 & & & & \\
\hline & & Phrynohyas Venulosa & 1 & 1 & & & 1 & \\
\hline & & Incilius sp. & & 1 & & & & \\
\hline & & Total & 3 & 8 & & 1 & 1 & 2 \\
\hline
\end{tabular}

\section{Reptiles}

\begin{tabular}{|c|c|c|c|c|c|c|c|c|}
\hline \multirow{2}{*}{ Clase } & \multirow{2}{*}{ N. común } & \multirow{2}{*}{ N. científico } & \multicolumn{6}{|c|}{ Lugar donde se encontró } \\
\hline & & & CM & CS & CMS & R1 & $\mathbf{R} \mathbf{2}$ & $\mathbf{R}_{3}$ \\
\hline \multirow{15}{*}{ Reptiles } & Perro sompopo & Sphaerodactylus millepunetatus & & & & & & 1 \\
\hline & Lagartija arborícola & Norops nebulosus & 4 & 3 & & & & \\
\hline & Anoli amarillo & Norops sericeus & & 1 & & 6 & & 4 \\
\hline & Basilisco común & Basiliscus basiliscus & 1 & & & & & \\
\hline & Basilisco marrón & Basiliscus vittatus & & 1 & & & & 2 \\
\hline & Culebra bejuquilla & Imantodes cenchoa & & & & & & 1 \\
\hline & $\begin{array}{l}\text { Culebra bejuquilla } \\
\text { cabezona }\end{array}$ & & & & & & & 1 \\
\hline & $\begin{array}{l}\text { Mabuya o esquinco } \\
\text { espalda dorada }\end{array}$ & Mabuya unimarginata & & & & & 1 & \\
\hline & $\begin{array}{l}\text { Lagartija corralera, } \\
\text { chombito }\end{array}$ & Sceloporus variabilis & 3 & 2 & 2 & & & \\
\hline & Anolis humilde. & Norops o anolis humilis & 2 & 6 & & & & 3 \\
\hline & Serpiente voladora & Spolotes pullatus & 1 & & & & & \\
\hline & Culebra chocoya flaca & leptophis nebulosus & & & & & & 1 \\
\hline & Barba amarrilla & bothrops asper & & & & 2 & & \\
\hline & Coral verdadero & Microrus nigrocinctus & & 1 & & & & \\
\hline & Lagartija, anolis & Anolis limifrons & & 3 & & & & \\
\hline
\end{tabular}




\begin{tabular}{|c|c|c|c|c|c|c|c|c|}
\hline \multirow{2}{*}{ Clase } & \multirow{2}{*}{ N. común } & \multirow{2}{*}{ N. científico } & \multicolumn{6}{|c|}{ Lugar donde se encontró } \\
\hline & & & CM & CS & CMS & R1 & $\mathbf{R 2}$ & $\mathbf{R}_{\mathbf{3}}$ \\
\hline \multirow{9}{*}{ Reptiles } & Ameiva arcoíris & Ameiva festiva & 7 & 9 & 2 & 9 & 3 & 5 \\
\hline & Lagartija dorada & Gymnopthalmus speciosus & 1 & & & & & \\
\hline & Zopilota & Clelia Clelia & & 1 & & & & \\
\hline & $\begin{array}{l}\text { Lagartija corredora } \\
\text { verdiazul }\end{array}$ & Aspidoscelis deppii & & 2 & & & & \\
\hline & Basilisco verde & Basiliscus plumifrons & & & & 1 & & \\
\hline & Anolis, Lagartija & Norops anolis polylepis & & & & 1 & & 4 \\
\hline & Lagartija anole & Norops limifrons o Slender & 5 & & 2 & & & 4 \\
\hline & & Norops spp & & & & & 1 & \\
\hline & & & 5 & 29 & 6 & 19 & 6 & 25 \\
\hline
\end{tabular}

\section{Lista de referencia}

Hidalgo. J. (2009). Estudio herpetológico comparativo en tres ecosistemas diferentes en la hacienda Cacao. Esmeraldas-Esmeraldas. Disponible en la web: http://repositorio.utpl.edu.ec/bitstream/123456789/3526/1/577X88.pdf [2011, 24 de Junio] 6op.

Matey. A, Zeledón. L, Orozco. L, Sampson. A, Chavarría. F (2010). Caracterización de la vegetación arbórea asociada al cacao y a fragmentos boscosos en fincas de Waslala, Nicaragua. Disponible en la web: http://www.catie.ac.cr/BancoMedios/ Documentos\%20PDF/pcc_caracteriza_vegeta.pdf [2011, 12 de Julio].

Kohler G. (2001): Anfibios y reptiles de Nicaragua.

Lennan. M (2009). Taxonomía, Biogeografía y Conservación de la Herpetofauna en Nicaragua. Disponible en: http//www.bio-nica.info/biblioteca/ Sunyer2oogHerpetofaunaNicaragua.pdf.

Soto Q. (2009). Centro Agronómico Tropical de Investigación y Enseñanza. Disponible en: http://www.orton.catie.ac.cr/repdoc/A4662E/A4662E.PDF

Soto, G. (2007-2008). Contribución al conocimiento del paisaje de cacaotales como hábitat para el mantenimiento de la diversidad de herpetofauna en Talamanca, Costa Rica. Disponible en. www.canacacao.org/modules/smartsection/visit.php?fileid $=45$ [2011,12 de abril] 